\title{
QUALIDADE DE VIDA DO PACIENTE SUBMETIDO À CIRURGIA VIDEOLAPAROSCÓPICA PARA TRATAMENTO PARA DOENÇA DO REFLUXO GASTROESOFÁGICO
}

\author{
Quality of life of patients undergoing surgery by videolaparoscopy for GERD treatment
}

Francisco José Cavalcante ANDRADE ${ }^{1,3}$, Eliete Rodrigues de ALMEIDA $^{3}$, Maria Teresa Botti Rodrigues dos SANTOS $^{3}$, Eurípedes SOARES-FILHO ${ }^{2}$,João Batista LOPES ${ }^{2}$, Roberto Cavalcante Veras e SILVA ${ }^{2}$

Trabalho realizado no ${ }^{1}$ Hospital Aliança Casamater e 2 Universidade Federal do Piauí, Terezina, PI, e ${ }^{3}$ Programa de Pós-Graduação da Universidade Cruzeiro do Sul, São Paulo, SP. Brasil.

DESCRITORES - Doença do refluxo gastroesofágico. Qualidade de vida. Cirurgia. Laparoscopia.
RESUMO - Racional - A doença do refluxo gastroesofágico é afecção crônica de elevada prevalência nos países ocidentais e com efeitos negativos sobre a qualidade de vida. O tratamento cirúrgico é indicado para pacientes que necessitam usar medicamentos ininterruptamente, intolerantes ao controle clínico prolongado e nas formas complicadas da doença. Objetivo - Avaliar a qualidade de vida de indivíduos submetidos à correção cirúrgica por videolaparoscopia, para tratamento da doença do refluxo gastroesofágico. Métodos - Foram avaliados 43 pacientes com idade média de 51,4 anos, de ambos os gêneros nos últimos dez anos, utilizando-se o questionário Escala de Qualidade de Vida Relacionada à Saúde para doença do refluxo gastroesofágico (1996), traduzido e validado para a língua portuguesa. Os dados foram analisados pelo programa estatístico Epi Info versão 3.5.1, com auxilio do teste de Duncan e do coeficiente de correlação de Pearson, fixando-se o valor de $5 \%$ para a hipótese de nulidade $(p \leq 0,05)$. Resultados - Mais de $50 \%$ dos participantes demonstraram boa qualidade de vida (escores $<5$ do questionário); 95,3\% indicaram satisfação com seu estado de saúde. Observou-se correlação positiva significativa entre a maioria das variáveis referentes à pirose e ao tempo após a operação $(p \leq 0,05)$. Conclusão - Os pacientes apresentaram boa qualidade de vida e elevado grau de satisfação com sua condição pós-operatória.

\section{Correspondência:}

Francisco José Cavalcante Andrade, e-mail: fjcavalcante@hotmail.com

Fonte de financiamento: não há Conflito de interesses: não há

Recebido para publicação: 06/03/2012 Aceito para publicação: 08/06/2012

HEADINGS - Gastroesophageal reflux disease. Quality of life. Surgery. Laparoscopy.
ABSTRACT - Background - Gastroesophageal reflux disease is a chronic disease of high prevalence in Western countries, with negative effects on quality of life. Surgery is indicated for patients with intolerance to continuous medication, prolonged treatment or control, or in complicated forms of the disease. Aim - To evaluate the quality of life of patients undergoing surgery by videolaparoscopy for gastroesophageal reflux disease treatment. Methods - Sample comprised 43 patients of both genders (mean age $=51.4$ years). For quality of life evaluation was made using the questionnaire Gastroesophageal Reflux Disease Health Related Quality of Life, translated and validated into Portuguese. Data were analyzed by Epi Info version 3.5.1, using Duncan test and Pearson's correlation coefficient, with $5 \%$ for null hypothesis ( $p \leq 0.05$ ). Results - Over $50 \%$ of participants showed good quality of life (scores $<5$ of the questionnaire), more than $90 \%$ indicated satisfaction with their health. A significant positive correlation between most variables related to heartburn and the time after surgery was observed ( $\leq \leq 0.05)$. Conclusion Patients presented good quality of life and high level of satisfaction with their postoperative condition. 
INTRODUÇÃO

A doença do refluxo gastresofágico (DRGE) é uma das afecções crônicas mais frequentes na prática médica, com elevada prevalência e morbidade, determinando limitações importantes no cotidiano de seus portadores, com prejuízo na qualidade de vida $(\mathrm{QV})^{1}$. Constitui-se em tema de crescente interesse para estudo, visto o elevado número de publicações na literatura. Não obstante, existe controvérsia sobre vários aspectos da afecção, do ponto de vista diagnóstico e terapêutico ${ }^{20}$.

Uma metanálise de estudos internacionais encontrou prevalência de $10-20 \%$ em países ocidentais e até $5 \%$ no mundo oriental, quando utiliza como critério diagnóstico para DRGE a presença de pirose pelo menos uma vez por semana, nos últimos 12 meses $^{8}$. No Brasil observou-se prevalência em torno de $11,8 \%$ para pirose uma vez por semana e $7,3 \%$ para pirose mais que uma vez por semana ${ }^{19}$.

Pode não haver critério padrão para definir a DRGE, uma vez que o limiar entre o refluxo fisiológico e patológico em última análise é arbitrário. Um consenso recente (consenso de Montreal) definiu a DRGE como "uma condição que se desenvolve quando o refluxo de conteúdos do estômago causa sintomas perturbadores e ou complicações"29.

As medidas terapêuticas buscam corrigir as alterações fisiopatológicas da doença, como melhorar a função motora do esôfago, elevar a pressão do EEI, acelerar o esvaziamento gástrico e minimizar o potencial danoso do conteúdo gástrico neutralizando ou mesmo suprimindo a agressão representada pelo ácido clorídrico do suco gástrico. Pode-se dividir a abordagem terapêutica em medidas comportamentais e farmacológicas, que deverão ser implementadas concomitantemente ${ }^{20}$. A grande dificuldade do tratamento clínico não consiste em controlar os sintomas, mas sim em manter os pacientes assintomáticos ao longo do tempo ${ }^{22}$.

Com o advento da cirurgia laparoscópica, houve maior anuência do paciente para a terapêutica cirúrgica. A menor morbidade pós-operatória nesta técnica não deve modificar suas indicações, porém esta opção tem-se tornado mais atrativa ${ }^{10}$. Contudo, sintomas pósoperatórios incluindo disfagia, dificuldade para eructar, flatulência e diarréia podem estar presentes, causando ansiedade e inquietação em pacientes e médicos ${ }^{17}$.

Desta forma, deve-se considerar o impacto da operação sobre a QV dos pacientes a ela submetidos. QV é assunto que vem sendo largamente debatido nas últimas décadas despertando o interesse da sociedade moderna. Tradicionalmente era conceito partilhado por cientistas sociais, filósofos e políticos, mas atualmente suscita crescente interesse de médicos e pesquisadores. A expressão QV na esfera da saúde está vinculada ao impacto do estado de saúde sobre a capacidade do indivíduo viver plenamente ${ }^{12}$. A Organização Mundial de Saúde reuniu especialistas, que definiram, em 1995, qualidade de vida como a "percepção do indivíduo de sua posição na vida no contexto da cultura e sistema de valores nos quais ele vive e em relação aos seus objetivos, expectativas, padrões e preocupações".

As mudanças nos paradigmas da assistência a saúde nas últimas décadas, colocam o indivíduo como um ser biopsicossocial, e não apenas como um organismo biológico que deve ser reparado. Conduzem a um cuidar mais humanizado, despertando para perspectiva da QV, levando em consideração a integralidade da pessoa humana4.

Assim, QV tem se tornado um conceito cada vez mais valorizado e utilizado em pesquisa clínica, bem como tem norteado decisões dos profissionais da saúde. A qualidade de vida relacionada à saúde (QVRS) tem sido utilizada para avaliar o impacto das doenças crônicas no cotidiano das pessoas e, também, para avaliar pacientes com a mesma doença, mas que apresentam critérios diferentes ao mesmo tratamento ${ }^{14}$.

O objetivo do presente estudo é analisar os resultados da utilização do questionário específico Gastroesophageal Reflux Disease Health Related Quality of Life (GERD-HRQL) para mensuração da QVRS de pacientes submetidos ao tratamento cirúrgico videolaparoscópico da DRGE.

\section{MÉTODOS}

Este estudo foi aprovado pelo Comitê de Ética e Pesquisa da Universidade Cruzeiro do Sul (UNICSUL) sob o número 159/2009. Todos os participantes leram, consentiram e assinaram o Termo de Consentimento Livre e Esclarecido.

Foram atendidos 43 indivíduos adultos, de ambos os gêneros, submetidos à operação por videolaparoscopia para tratamento da DRGE, no hospital Casamater, na cidade de Teresina, PI, Brazil nos últimos 10 anos.

Os critérios de inclusão foram: pacientes de ambos os gêneros, acima de 18 anos de idade submetidos à operação para tratamento de DRGE nos últimos dez anos operados pela mesma equipe cirúrgica utilizando a técnica de Nissen-Rossetti (fundoplicatura de $360^{\circ}$ ).

Foram excluídos os pacientes submetidos à outras operações abdominais após o tratamento cirúrgico da DRGE.

Todos foram avaliados mediante a utilização de questionários, a partir de entrevistas individuais realizadas em ambiente apropriado. Os questionários foram:

1) Dados Sócio-Demográficos (elaborado especialmente para este estudo) e 2) GERD-HRQL em português (Escala de Qualidade de Vida Relacionada à Saúde para Doença do Refluxo Gastroesofágico DRGE-QVRS). 


\section{Análise e interpretação dos dados}

A criação do banco de dados e as análises estatísticas foram realizadas com auxílio do programa Epi Info versão 3.5.1.

Os dados foram analisados de forma descritiva através de frequência relativa (\%) e absoluta (n) para as variáveis categóricas. As variáveis quantitativas e os escores das variáveis de mensuração da escala de QV foram expressos através de média, desvio-padrão, valores mínimos e máximos.

Para comparar as médias das diversas variáveis estudadas, em função da faixa etária e do tempo pósoperatório e para verificar a relação linear significativa entre os parâmetros do questionário GERD-HRQL, foi utilizado o coeficiente de correlação de Pearson. Fixouse $o$ valor de $5 \%$ para as hipóteses de nulidade $(p \leq$ $0,05)$.

\section{RESULTADOS}

A média de idade dos pacientes foi de 51,4 anos, com variação de 23-81 anos (Tabela 1). Apenas um paciente $(2,3 \%)$ era sem estudos, enquanto $41,9 \%$ tinham ensino superior completo. Com relação à etnia, de acordo com critérios do IBGE onde o indivíduo diz a que classe pertence, encontrou-se $67,4 \%$ de brancos e $32,6 \%$ de pardos (Tabela 1 ).

TABELA 1 - Distribuição absoluta e relativa da amostra, segundo características sócio-demográficas

\begin{tabular}{|c|c|}
\hline Características & Valores \\
\hline \multicolumn{2}{|l|}{ Idade } \\
\hline $\begin{array}{l}\text { Média e faixa de variação (em anos) } \pm \\
\text { desvio-padrão }\end{array}$ & $51,8(23-81) \pm 12,5(\mathrm{DP})$ \\
\hline Gênero & $\mathrm{N}(\%)$ \\
\hline Feminino & $21(48,8)$ \\
\hline Masculino & $22(51,2)$ \\
\hline Escolaridade & $\mathrm{N}(\%)$ \\
\hline Sem Estuos & $1(2,3)$ \\
\hline Ensino fundamental & $7(16,3)$ \\
\hline Ensino médio & $13(30,2)$ \\
\hline Ensino superior & $18(41,9)$ \\
\hline Ensino superior Incompleto & $4(9,3)$ \\
\hline Etnia & $\mathrm{N}(\%)$ \\
\hline Branca & $29(67,4)$ \\
\hline Parda & $14(32,6)$ \\
\hline Negra & - \\
\hline Amarela & - \\
\hline Estado Civil & $\mathrm{N}(\%)$ \\
\hline Solteiro & $8(18,6)$ \\
\hline União estável & $33(76,7)$ \\
\hline Viúvo e separado & 1 de cada $(2,3 \times 2)$ \\
\hline
\end{tabular}

O escore no questionário DRGE-QVRS foi calculado pela soma das cifras assinalados pelos pacientes, variando entre 0 e 50 ( 0 a melhor resposta e 50, a pior). Entre os resultados obtidos, observouse predominância de escores baixos, onde o escore mínimo (zero) estava presente em $16,3 \%$ dos pacientes.
Vale ainda ressaltar que mais da metade dos pacientes apresentou escores abaixo de 5 e somente um exibiu escore de 18, máximo alcançado neste estudo (Figura 1).

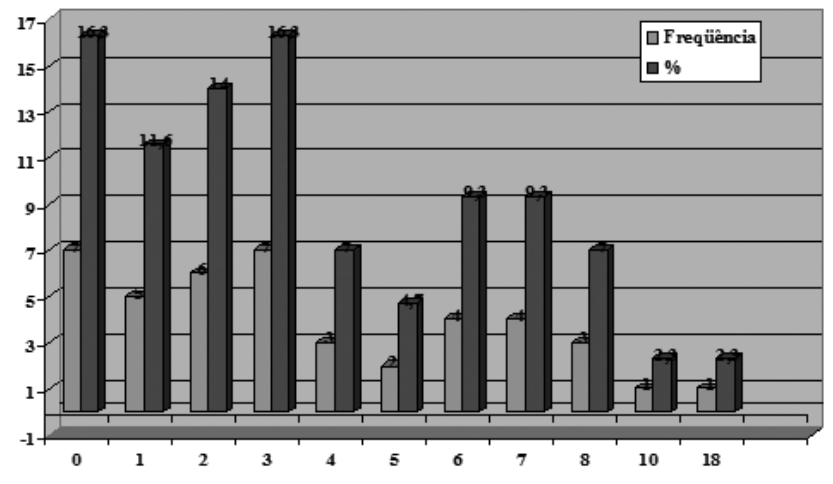

FIGURA 1 - Distribuição relativa e absoluta da amostra, segundo escores de qualidade de vida

As Tabelas 2 e 3 apresentam as médias dos escores quanto aos parâmetros avaliados, a faixa etária e tempo decorrido da operação.

TABELA 2 - Distribuição da amostra segundo a média dos parâmetros avaliados em função da faixa etária

\begin{tabular}{|c|c|c|c|c|c|}
\hline \multirow{2}{*}{ Parâmetros } & \multicolumn{4}{|c|}{ Faixa etária (anos) } & \multirow{2}{*}{ QV } \\
\hline & [23-38] & [38-53] & [53-68] & [68-83] & \\
\hline $\begin{array}{l}\text { Tempo de operação } \\
\text { (anos) }\end{array}$ & $32,00 a$ & $43,85 b$ & $59,42 c$ & $73,00 d$ & 9,2 \\
\hline $\begin{array}{l}\text { O quanto ruim é a } \\
\text { azia (escore) }\end{array}$ & $1,35 b$ & $0,00 b$ & $1,16 b$ & $6,03 a$ & 228,1 \\
\hline $\begin{array}{l}\text { Azia quando deitado } \\
\text { (escore) }\end{array}$ & $0,00 b$ & $0,00 b$ & $1,05 b$ & $6,03 a$ & 241,8 \\
\hline $\begin{array}{l}\text { Azia quando em pé } \\
\text { (escore) }\end{array}$ & $1,35 \mathrm{a}$ & $0,00 a$ & $1,05 a$ & $2,71 a$ & 279,5 \\
\hline $\begin{array}{l}\text { Azia depois das } \\
\text { refeições (escore) }\end{array}$ & $1,35 a$ & $0,00 a$ & $0,77 a$ & $2,71 a$ & 313,1 \\
\hline $\begin{array}{l}\text { Azia altera o que } \\
\text { come (escore) }\end{array}$ & $0,00 \mathrm{~b}$ & $0,44 b$ & $0,39 b$ & $6,56 a$ & 262,6 \\
\hline $\begin{array}{l}\text { Azia faz acordar } \\
\text { durante o sono } \\
\text { (escore) }\end{array}$ & $0,00 a$ & $0,00 a$ & $0,66 a$ & $0,00 a$ & 470,6 \\
\hline $\begin{array}{l}\text { Tem dificuldade em } \\
\text { engolir (escore) }\end{array}$ & $3,97 a$ & $2,46 a$ & $4,03 a$ & $2,71 a$ & 118,1 \\
\hline $\begin{array}{l}\text { Tem dor ao engolir } \\
\text { (escore) }\end{array}$ & $0,00 a$ & $1,25 \mathrm{a}$ & $1,43 a$ & $3,32 a$ & 233,7 \\
\hline $\begin{array}{l}\text { Tem inchaço ou } \\
\text { sensação de gases } \\
\text { (escore) }\end{array}$ & $4,37 a$ & $4,73 a$ & $5,23 a$ & $2,71 a$ & 87,6 \\
\hline $\begin{array}{l}\text { Toma medicação e } \\
\text { isto afeta a vida diária } \\
\text { (escore) }\end{array}$ & $1,66 a$ & $0,88 a$ & $1,37 a$ & $1,91 a$ & 208,4 \\
\hline Escore & $9,78 b$ & $6,79 b$ & $10,57 a b$ & $16,55 a$ & 56,3 \\
\hline
\end{tabular}

Médias seguidas da mesma letra na inha não diferiram entre si pelo teste de Duncan $(p>0,05)$

A última questão do GERD-HRQL avaliou a percepção do paciente quanto ao seu estado de saúde atual em seis níveis de satisfação. As respostas muito satisfeito e satisfeito foram assinaladas, respectivamente, por $25(58,1 \%)$ e $16(37,2 \%)$ dos pacientes estudados. 
TABELA 3 - Distribuição da amostra segundo a média dos parâmetros avaliados em função do tempo decorrido após a operação

\begin{tabular}{|c|c|c|c|c|c|c|}
\hline \multirow{2}{*}{ Parâmetros } & \multicolumn{5}{|c|}{ Tempo decorrido após a operação (em anos) } & \multirow{2}{*}{ QV(\%) } \\
\hline & {$[0,5-2,5]$} & {$[2,5-4,5]$} & {$[4,5-6,5]$} & {$[6,5-8,5]$} & {$[8,5-0,5]$} & \\
\hline $\begin{array}{l}\text { O quanto ruim é a } \\
\text { azia (escore) }\end{array}$ & $0,250 a$ & $0,200 a$ & $0,385 a$ & $0,286 a$ & $0,400 a$ & 267,2 \\
\hline $\begin{array}{l}\text { Azia quando } \\
\text { deitado (escore) }\end{array}$ & $0,250 a$ & $0,200 a$ & $0,231 a$ & $0,286 a$ & $0,200 a$ & 309,1 \\
\hline $\begin{array}{l}\text { Azia quando em pé } \\
\text { (escore) }\end{array}$ & $0,000 \mathrm{a}$ & $0,200 a$ & $0,308 \mathrm{a}$ & $0,143 a$ & $0,400 a$ & 294,4 \\
\hline $\begin{array}{l}\text { Azia depois das } \\
\text { refeições (escore) }\end{array}$ & $0,000 \mathrm{a}$ & $0,200 a$ & $0,307 a$ & $0,000 a$ & $0,400 a$ & 321,2 \\
\hline $\begin{array}{l}\text { Azia altera o que } \\
\text { come (escore) }\end{array}$ & $0,250 a$ & $0,100 a$ & $0,462 a$ & $0,000 a$ & $0,000 a$ & 360,5 \\
\hline $\begin{array}{l}\text { Azia faz acordar } \\
\text { durante sono } \\
\text { (escore) }\end{array}$ & $0,000 a$ & $0,000 a$ & $0,000 a$ & $0,143 a$ & $0,400 a$ & 468,3 \\
\hline $\begin{array}{l}\text { Tem dificuldade em } \\
\text { engolir (escore) }\end{array}$ & $0,750 \mathrm{ab}$ & $1,100 \mathrm{ab}$ & $1,385 a$ & $0,000 c$ & $0,200 a b$ & 110,1 \\
\hline $\begin{array}{l}\text { Tem dor ao engolir } \\
\text { (escore) }\end{array}$ & $0,143 a$ & $0,600 a$ & $0,615 a$ & $0,143 a$ & $0,143 a$ & 230,9 \\
\hline $\begin{array}{l}\text { Inchaço / sensação } \\
\text { gases (escore) }\end{array}$ & $1,375 a$ & $1,000 a$ & $1,307 a$ & $1,000 a$ & $1,200 \mathrm{a}$ & 98,1 \\
\hline $\begin{array}{l}\text { Toma medicação } \\
\text { afeta a vida diária } \\
\text { (escore) }\end{array}$ & $0,125 a$ & $0,100 a$ & $0,153 a$ & $0,857 a$ & $0,200 a$ & 211,5 \\
\hline Escore & $3,000 a$ & $3,700 a$ & $5,154 a$ & $2,174 a$ & $3,400 a$ & 93,4 \\
\hline
\end{tabular}

Médias seguidas da mesma letra na linha não diferiram entre si pelo teste de Duncan $(p>0,05)$

Notou-se que a maioria dos pacientes muito satisfeitos concentravam-se entre dois e sete anos decorridos da operação (Figura 2).

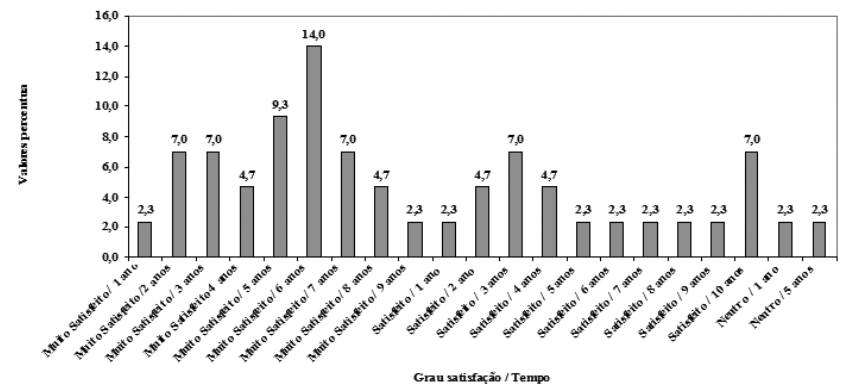

FIGURA 2 - Distribuição da amostra segundo grau de satisfação em função do tempo após a operação
A Tabela 4 apresenta a análise estatística entre as variáveis estudadas e o desfecho - qualidade de vida, utilizando-se o teste de correlação Pearson.

\section{DISCUSSÃO}

As últimas décadas contemplaram um momento importante nos estudos referentes ao manejo da DRGE, decorrentes dos incrementos diagnósticos e terapêuticos. Fator relevante a ser considerado neste manejo é a crescente aceitação de que a QV dos pacientes é amplamente afetada pelos sintomas relacionados à doença, tornando sua avaliação importante na condução dos pacientes6.

Apesar do advento dos inibidores de bomba de prótons (IBP), alguns pacientes continuam a experimentar sintomas persistentes de DRGE. Alternativas não-cirúrgicas para tratamento desses pacientes são atualmente limitadas ${ }^{18,20}$.

O presente estudo apresentou os escores da QVRS de pacientes submetidos ao tratamento cirúrgico para DRGE, a partir da utilização do instrumento de GERDHRQL.

Algumas limitações devem ser ressaltadas, notadamente relacionadas às peculiaridades metodológicas relativas ao desenho do estudo, o qual não permite confrontar a QV passada e atual de cada participante da amostra, por não se tratar de estudo longitudinal.

Os escores totais baixos para o GERD-HRQL, obtidos na amostra, indicaram boa qualidade de vida, sugerindo que o tratamento cirúrgico foi eficaz para os pacientes estudados. Devido à particularidade de delineamento deste estudo, não é cabível afirmar que o tratamento cirúrgico promoveu melhora na qualidade de vida dos indivíduos pesquisados. A qualidade de vida aqui relatada alude-se ao momento da coleta dos dados.

As alterações no estilo de vida impostas pela necessidade de controle dos sintomas, como

TABELA 4 - Análise estatística das variáveis estudadas (DRGE-HRQL), segundo teste de correlação de Pearson

\begin{tabular}{|c|c|c|c|c|c|c|c|c|c|c|c|c|c|}
\hline & Idade & $\begin{array}{c}\text { Tempo } \\
\text { operação }\end{array}$ & $\begin{array}{l}\text { Quanto } \\
\text { aazia é ruim }\end{array}$ & $\begin{array}{l}\text { Azia } \\
\text { deitado }\end{array}$ & $\begin{array}{l}\text { Azia } \\
\text { em pé }\end{array}$ & $\begin{array}{l}\text { Azia apos } \\
\text { refeições }\end{array}$ & $\begin{array}{l}\text { Azia altera o } \\
\text { que come }\end{array}$ & $\begin{array}{l}\text { Azia durante } \\
\text { sono }\end{array}$ & $\begin{array}{l}\text { Dificuldade } \\
\text { de engolir }\end{array}$ & $\begin{array}{l}\text { Dor ao } \\
\text { engolir }\end{array}$ & $\begin{array}{c}\text { Inchaço / } \\
\text { gases }\end{array}$ & $\begin{array}{c}\text { Toma } \\
\text { medicação }\end{array}$ & Escore \\
\hline Idade & - & - & - & - & - & - & - & - & - & - & - & - & - \\
\hline Tempo de operação & 0,321 & - & - & - & - & - & - & - & - & - & - & - & - \\
\hline Quanto aazia é ruim & 0,321 & 1,002 & - & - & - & - & - & - & - & - & - & - & - \\
\hline Azia deitado & 0,442 & 0,762 & 0,761 & - & - & - & - & - & - & - & - & - & - \\
\hline Azia em pé & $0,21 \mathrm{NS}$ & 0,902 & 0,901 & 0,572 & - & - & - & - & - & - & - & - & - \\
\hline Azia aposrefeições & $0,18 \mathrm{NS}$ & 0,802 & 0,811 & 0,442 & 0,942 & - & - & - & - & - & - & - & - \\
\hline Azia altera o que come & 0,391 & 0,451 & 0,451 & 0,532 & $0,24 \mathrm{NS}$ & $0,27 \mathrm{NS}$ & - & - & - & - & - & - & - \\
\hline Azia durante sono & $0,16 \mathrm{NS}$ & $0,16 \mathrm{NS}$ & 0,16 NS & 0,482 & $0,13 N S$ & $0,07 \mathrm{NS}$ & $-0,07 \mathrm{NS}$ & - & - & - & - & - & - \\
\hline Dificuldade de engolir & $0,01 \mathrm{NS}$ & $0,22 \mathrm{NS}$ & $-0,22 \mathrm{NS}$ & $-0,311$ & $0,16 \mathrm{NS}$ & $-0,11 \mathrm{NS}$ & $-0,27 \mathrm{NS}$ & $-0,19 N S$ & - & - & - & - & - \\
\hline Dor aoengolir & $0,25 \mathrm{NV}$ & $0,09 \mathrm{NS}$ & $0,09 \mathrm{NS}$ & $0,14 \mathrm{NS}$ & $0,10 \mathrm{NS}$ & $0,12 \mathrm{NS}$ & $0,22 \mathrm{NS}$ & $0,09 \mathrm{NS}$ & 0,381 & - & - & - & - \\
\hline Inchaço /gases & $0,03 \mathrm{NS}$ & $0,09 \mathrm{NS}$ & $-0,09 N S$ & $-0,27 \mathrm{NS}$ & $0,12 \mathrm{NS}$ & $-0,06 \mathrm{NS}$ & $0,01 \mathrm{NS}$ & $-0,25 \mathrm{NS}$ & $0,19 \mathrm{NS}$ & $0,19 \mathrm{NS}$ & - & - & - \\
\hline Toma medicação & 0,07 NS & $0,12 \mathrm{NS}$ & $0,12 \mathrm{NS}$ & $0,17 \mathrm{NS}$ & $0,10 \mathrm{NS}$ & 0,02NS & $0,08 \mathrm{NS}$ & $0,09 \mathrm{NS}$ & $-0,12 \mathrm{NS}$ & $0,23 \mathrm{NS}$ & 0,26NS & - & - \\
\hline Escore & 0,321 & 0,591 & 0,592 & 0,482 & 0,552 & 0,522 & 0,382 & 0,09 NS & 0,321 & 0,501 & 0,491 & 0,402 & - \\
\hline
\end{tabular}


modificações nos hábitos alimentares, elevação da cabeceira da cama e um dormir desconfortável, podem afetar a sensação de bem estar físico e a capacidade de convivência dos pacientes alterando suas relações sociais e afetando seu status psicológico, podendo levar a impacto negativo sobre sua qualidade de vida. Todos estes aspectos de bem estar podem ser melhorados pela operação ${ }^{6}$.

A perspectiva do paciente diante dela é livrar-se dos sintomas da DRGE e não adquirir efeitos colaterais incômodos. A QV será melhorada pelo desaparecimento dos sintomas de refluxo e a ausência de novos sintomas como disfagia. Além disto, deve ser possível regressar à normalidade dos hábitos alimentares. Embora a operação possa determinar melhorias anatômicas e funcionais no esfincter esofágico inferior, ela pode não ser satisfatória para o controle dos sintomas da DRGE, quando avaliada sobre o ponto de vista dos pacientes.

No grupo estudado, a idade dos pacientes variou de 23 a 81 anos, com média de 51,8 anos, que não se mostrou diferente dos dados da literatura, cuja média de idade de pacientes com DRGE varia de 39,6 a $59,75,19,21$.

Quanto ao gênero, observou-se certa discordância com a população de pacientes com DRGE descrita em vários trabalhos da literatura, com maior prevalência de mulheres e variação de $57-61,8 \%{ }^{19,21}$.

$\mathrm{Na}$ amostra deste estudo, encontrou-se $48,8 \%$ do gênero feminino, dados semelhantes aos de Ciovica et al. ${ }^{5}$, em estudo sobre QV apos operação laparoscópica anti-refluxo, com prevalência de $48,4 \%$ do gênero feminino. A DRGE não assume clara preferência por sexo, como já foi demonstrado na literatura, apesar da associação conhecida entre sintomas de DRGE e gravide $z^{8}$. As diferenças encontradas, podem ser atribuídas à maior probabilidade de diagnóstico entre pacientes do gênero feminino com níveis mais elevados de cuidados médicos.

Sonnenberg e El-Serag ${ }^{24}$ referiram que as formas mais severas da DRGE caracterizadas por esofagite erosiva e úlceras esofágicas são mais comuns entre os homens.

Com relação à escolaridade, observou-se predominância de indivíduos com ensino superior (41,9\%), baixa proporção de indivíduos com ensino fundamental $(16,3 \%)$ e apenas uma pessoa sem estudos (2,3\%). Estes dados estão em desacordo com um estudo populacional brasileiro realizado no Rio Grande do Sul, o qual demonstrou maior prevalência de DRGE em faixa de baixa escolaridade ${ }^{21}$. Contudo estes resultados não causaram estranheza e poderiam ser explicados, pelo fato de que o trabalho foi realizado em unidade hospitalar da capital do estado, que atende planos de saúde, portanto população com melhor posição socioeconômica, onde se espera melhor escolaridade.

Como mencionado anteriormente, para avaliar a QVRS dos pacientes, foi utilizado o questionário DRGEQVRS, onde o escore é calculado pela soma das notas assinaladas pelos pacientes. A maior parte $(69,9 \%)$ apresentou escores baixos ( 0 a 5 ) indicando eficácia do tratamento estabelecido no que se refere à $\mathrm{QV}$ dos pacientes estudados. Na literatura especializada a maioria dos trabalhos faz comparação entre escores de QV em momentos pré e pós-operatórios, assim como checagens com tratamento clínico prolongado, indicando melhora após o tratamento cirúrgico. Isso sugere que esta modalidade terapêutica é melhor que a terapia medicamentosa quando se considera qualidade de vida e satisfação dos pacientes ${ }^{5,6,15,27}$.

Conquanto este estudo não confrontasse os dados pré e pós-tratamento cirúrgico e devido à característica de seu delineamento, não é permitido afirmar se houve melhora na QV dos pacientes estudados. Os dados apontam boa qualidade de vida da amostra estudada no ato do questionamento. Vários autores têm demonstrado, claramente, melhora na QV após a operação, embora admitindo a persistência de alguns sintomas, como plenitude e distensão abdominal, os quais, em geral, são bem tolerados pelos pacientes ${ }^{2,7,26}$.

Neste estudo, ao analisar a resposta para a nona pergunta do questionário, sobre inchaço ou sensação de gases, observou-se anuência com a afirmação da literatura sobre a presença dos sintomas, com tolerância relativamente boa por parte dos pacientes.

A última questão do GERD-HRQL avaliou a percepção do paciente quanto ao seu estado de saúde atual em seis níveis de satisfação. Entre os casos estudados, $58,1 \%$ indicaram estar muito satisfeitos, $37,2 \%$ estavam satisfeitos e $4,7 \%$ referiram o grau de satisfação como neutro, nenhum paciente assinalou as respostas: insatisfeito, muito insatisfeito ou incapacitado. De forma semelhante Tucker et al. ${ }^{27}$ reportaram $73,1 \%$ pacientes completamente satisfeitos, $22,8 \%$ pouco satisfeitos e apenas $5,3 \%$ insatisfeitos com a cirurgia.

Quando avaliadas as respostas dos pacientes em função do tempo decorrido da operação, foi observado que a maioria muito satisfeita concentravam-se entre dois e sete anos decorridos da operação. Estes dados vão ao encontro de Hamdy et al. ${ }^{15}$ que evidenciaram abrandamento de sintomas, como odinofagia, disfagia e distensão gasosa, ao longo do tempo pós-operatório, melhorando o grau de satisfação do paciente com o procedimento.

As questões 1-6 do GERD-HRQL trataram da pirose, sintoma típico da DRGE que afeta negativamente a QV dos pacientes. Neste estudo, as respostas assinaladas variaram de zero (sem sintomas) a quatro (sintomas afetam atividades diárias). A grande maioria assinalou zero (sem sintomas), o que poderia ser explicado pela efetividade da operação no controle dos sintomas, com consequente melhora da QV. Ao comparar as médias destas questões, em função do tempo decorrido do pós-operatório, estas não diferiram entre si pelo teste de Duncan a $5 \%$ de probabilidade de diferenças entre as médias. 
Os itens 7-9 do instrumento utilizado no estudo referiram-se aos sintomas de disfagia, odinofagia e meteorismo, considerados efeitos colaterais do procedimento. Nesta série, estes itens são as principais respostas que elevam os escores finais, diminuindo QV e grau de satisfação dos pacientes. Porém, como mencionado anteriormente, estes sintomas são geralmente bem tolerados pelos pacientes e apresentam melhoria ao longo do tempo após a operação conforme exposto na literatura ${ }^{2,15,26}$. Observou-se diferença apenas nas médias da pergunta sobre disfagia, com tendência de diminuição deste sintoma com tempo mais prolongado.

Spechler et al. ${ }^{25}$ comparando fundoplicatura laparotômica e tratamento medicamentoso, mostraram que dez anos após a operação, 62\% dos pacientes usavam drogas anti-refluxo regularmente. Contudo, $86 \%$ estavam satisfeitos com os resultados do procedimento.

O item 10 do questionário GERD-HRQL, tratou do uso de medicação. Neste estudo, $79,1 \%$ dos pacientes assinalaram zero (sem sintomas) para esta questão, 18,6\% marcaram 1 (nota-se sintomas, mas não incomodo) e apenas um paciente apontou nota 3 (sintomas incômodos todos os dias), revelando baixa necessidade do uso de medicação contínua e, consequentemente, pequeno impacto sobre sua QV. Dallemagne et al. ${ }^{7}$ relataram que menos de $10 \%$ dos pacientes necessitavam usar medicação antirefluxo de forma contínua, dez anos após a operação laparoscópica, dado mais aproximado ao que se encontrou nas respostas para o item 10.

Observou-se correlação positiva significativa entre a maioria das variáveis que se referiam à pirose e ao tempo após a operação. Por outro lado houve correlação negativa, porém não significativa, entre a 'dificuldade de engolir e empachamento' com 'pirose e tempo decorrido da operação', sugerindo que o melhor controle da pirose poderia determinar maiores efeitos colaterais pós-operatórios, como disfagia, sensação de plenitude e certa tendência a recorrência de azia ao longo do tempo.

A QV é ponto importante, que deve ser analisado ao indicar o tratamento para DRGE. Habitualmente, as pessoas portadoras dessa entidade clínica têm QV ruim quando comparados com indivíduos saudáveis, justificando a satisfação com relação à operação, apesar de alguns sintomas ainda presentes, conforme relatados pelos pacientes deste estudo e em outros já publicados.

$O$ instrumento de avaliação utilizado neste estudo GERD-HRQL, apresentou fácil entendimento pelos pacientes, baseado em escala de sintomas típicos da DRGE, utilizando-se o sintoma pirose, considerando suas influências sobre alimentação, sono e a necessidade do uso de medicações como marcadores das repercussões sobre a QV dos pacientes com DRGE. O questionário apresenta também itens sobre flatulência, distensão abdominal e disfagia, que permitem avaliar pacientes submetidos à operação anti-refluxo com possíveis efeitos colaterais pós-operatórios que poderiam repercutir negativamente sobre a QV. Observou-se a falta de questões sobre manifestações extra-esofágicas (globo faríngeo, halitose, asma, tosse e rouquidão), que podem afetar a qualidade de vida dos pacientes. Contudo estes não são sintomas típicos da DRGE.

Instrumento específico de avaliação da QV aqui utilizado foi constituído e validado por Velanovich ${ }^{30}$ baseado em uma escala de sintomas típicos para a DRGE. Ele foi traduzido e validado para a língua portuguesa por Pereira22.

\section{CONCLUSÃO}

Os pacientes nos últimos 10 anos, apresentaram baixos escores referentes ao GERD-HRQL (pósoperatório), apresentando boa QV. Notou-se menor queixa do sintoma 'disfagia pós-operatória' em pacientes com mais tempo de pós-operatório. $\mathrm{Na}$ faixa etária mais avançada (68 a 83 anos) observaramse escores mais elevados, com menor impacto do tratamento cirúrgico sobre a qualidade de vida ao longo dos anos. Independentemente do escore do GERD-HRQL observado, os pacientes apresentaram elevado grau de satisfação com sua condição pósoperatória.

\section{REFERÊNCIAS}

1. Associação Interamericana de Gastroenterologia; Colégio Brasileiro de Cirurgia Digestiva; Federação Brasileira de Gastroenterologia; Sociedade Brasileira de Endoscopia Digestiva; Sociedade Brasileira de Motilidade Digestiva. II Consenso Brasileiro da Doença do Refluxo Gastroesofágico. São Paulo (Brasil); 2003.

2. Balci $D$, Turkcapar AG. Assessment of quality of life after laparoscopic Nissen fundoplication in patients with gastroesophageal reflux disease. World J Surg 2007; 31: 116-21.

3. Borgaonkar MR, Irvine EJ. Quality of life measurement in gastrointestinal and liver disorders. Gut 2000;47:444-54.

4. Ciconelli RM, Ferraz MB, Santos W, Meinão I, Quaresma MR. Tradução para língua portuguesa e validação do questionário genérico de avaliação de qualidade de vida SF-36 (Brasil SF-36). Ver Brás Reumatol.1999; 39(3):143-50.

5. Ciovica R, Gadenstätter M, Klingler A, Neumayer C, Schwab GP. Laparoscopic antireflux surgery provides excellent results and quality of life in gastroesophageal reflux disease patients with respiratory symptoms. J Gastrointest Surg.2005; 9(5): 633-7.

6. Ciovica R, Gadenstätter M, Klingler A, Wolfgang L, Riedl O, Schwab GP. Quality of Life in GERD Patients: Medical Treatment Versus Antireflux Surgery. J Gastrointest Surg.2006; 10(7): 934-9.

7. Dallemagne B, Weerts J, Markiewicz S, Dewandre JM, Wahlen C, Monami B, Jehaes C. Clinical Results of Laparoscopic Fundoplication at Ten Years After Surgery. Surg Endosc. 2006; 20(1):159-65.

8. Dent J, El-Serag HB, Wallander MA|, Johansson S. Epidemiology of gastro-oesophageal reflux disease: a systematic review. Gut 2005;54;710-7.

9. De Souza-Cury M, Ferrari AP, Ciconelli R, Ferraz MB, Moraes-Filho $J P$. Evaluation of health-related quality of life in gastroesophageal reflux disease patients before and after treatment with pantoprazole. Dis Esophagus. 2006;19:289-93. 
10. De Vault KR, Castell DO. Updated guidelines for the treatment of gatroesophageal refl ux disease. Am J Gastroenterol. 2005; 100:190-200.

11. Donnellan C, Sharma N, Preston C, Moayyedi P. Medical treatments for the maintenance therapy of refl ux oesophagitis and endoscopic negative refl ux disease (Cochrane Review). In: The Cochrane Library, Issue 2. Oxford: Update Software; 2005.

12. Fleck MPA, Leal OF, Louzada S, Xavier M, Chachamovich E, Vieira $G$, et al. Desenvolvimento da versão em português do instrumento de avaliação de qualidade de vida da OMS (WHOQOL-100). Rev Bras Psiquiatr. 1999; 21 (1): 19-28.

13. Fornari F, Gruber AC, Lopes AB, Cecchetti D, Barros SGS. Questionário de sintomas na doença do refluxo gastroesofágico. Arq Gastroenterol. 2004; 41 (4): 263-7.

14. Guyatt GH, Feeny DH, Patrick DL. Measuring Health-related Quality of Life. Annals of Internal Medicine. 1993; 118 (8): 622-9.

15. Hamdy E, El-Raouf AA, El-Hemaly M, Salah T, El-Hanafy E, Mostafa M, et al. Quality of life and patient satisfaction 3 months and 3 years after laparoscopic Nissen's fundoplication. Saudi J Gastroenterol. 2008; 14(1): 24-7.

16. Irvine EJ. Quality of life assessment in gastro-oesophageal reflux disease. Gut.2004; 53: 35-9.

17. Klaus A, Hinder RA, De Vault KR, Achem SR. Bowel dysfunction after laparoscopic antireflux surgery: incidence, severity, and clinical course. Am J Med. 2003; 114:6-9.

18. Lehmann A. GABAB receptors as drug targets to treat gastroesophageal reflux disease. Pharmacol Ther. 2009; 122(3): 239-45.

19. Moraes-Filho JPP, Chinzon D, Eisig JN, Hashimoto CL, Zaterka S. Prevalence of heartburn And gastroesophageal Reflux disease in the urban Brazilian population. Arq Gastroenterol. 2005; 42(2): 122-7.

20. Nasi A, Moraes-Filho JPP, Cecconello I. Doença do refluxo gastroesofágico: revisão ampliada. Arq Gastroenterol. 2006; 43 (4): 334-40.

21. Oliveira SS, Santos IS, Silva JFP, Machado EC. Prevalência e fatores associados à doença do refluxo gastroesofágico. Arq Gastroenterol. 2005; 42(2): 116-21.
22. Pereira GIN, Costa CDS, Geocze L, Borim AA, Ciconelli RM, Camacho-Lobato $L$. Tradução e validação para a língua portuguesa (brasil) de instrumentos específicos para avaliação de qualidade de vida na doença do refluxo gastroesofágico. Arq Gastroenterol. 2007; 44 (2): 168-77.

23. Pose AC, Reyes L, Saona MG, Umpierre V. Manometria esofágica en pacientes con disfagia, reflujo gastroesofágico y dolor torácico no cardíaco. Utilidad diagnóstica. Ver Med Urug. 2009; 25: 34-44.

24. Sonnenberg A, El-Serag HB. Clinical epidemiology and natural history of gastroesophageal reflux disease. Yale J Biol Med 1999; 72(2-3): 81-92.

25. SpeSpechler SJ, Lee E, Ahnen D, Goyal RK, Hirano I, Ramirez F, Raufman JP, Sampliner R, Schnell T, Sontag S, Vlahcevic ZR, Young $R$, Williford W.. Long-term outcome of medical and surgical therapies for gastroesophageal reflux disease: Follow-up of a randomized controlled trial. JAMA 2001; 285(18): 2331-8.

26. Teixeira JPA, Mosquera V, Flores A. Long-term outcomes of quality of life after laparoscopic nissen fundoplication. HepatoGastroenterology 2009; 56: 80-4.

27. Tucker LE, Blatt C, Richardson NL, Richardson DT, Cassat JD, Riechers TB. Laparoscopic Nissen fundoplication in a community hospital: Patients satisfaction survey. Southern Medical Journal 2005; 98(4): 441-3.

28. Tutuian R, Castell DO. Gastroesophageal reflux monitoring: $\mathrm{pH}$ and impedance. GI motility online [periódico na internet]. 2006 [acesso em: 2009 Set 14]. Disponível em: http://www.nature.com/ gimo/contents/index.html

29. Vakil N, van Zanten SV, Kahrilas P, Dent J, Jones R; Global Consensus Group. The Montreal Definition and Classification of Gastroesophageal Reflux Disease: A Global Evidence-Based Consensus. Am J Gastroenterol. 2006; 101:1900-20.

30. Velanovich V, Vallance SR, Gusz JR, Tapia FV, Harkabus MA. Quality of life scale for gastroesophageal reflux disease. J Am Coll Surg 1996; 183(3): 217-24. 